\title{
FIR Approximation of GTD Filter Banks and their Multiresolution Optimality
}

\author{
Aravind Illa \\ Department of Electronics and communication Engineering \\ National Institute of Technology Calicut \\ Kerala 673601, India
}

\author{
Elizabeth Elias \\ Department of Electronics and communication Engineering \\ National Institute of Technology Calicut \\ Kerala 673601, India
}

\begin{abstract}
The theory and design of signal adapted filter banks in the coding gain objective as well as the multiresolution objective are of great interest in many signal processing applications. The role of the generalized triangular decomposition (GTD) filter banks in optimizing perfect reconstruction filter banks has been proposed recently by Ching-Chih Weng et al. They have proposed the GTD filter bank as a subband coder for optimizing the theoretical coding gain. In this paper, we show that the design of the GTD filter bank via the singular value decomposition (SVD) will be reduced to the principal component filter bank (PCFB) and it gives optimal performance in the multiresolution objective. The FIR approximation of the optimal GTD filter banks is also discussed in this paper. This is done by using the iterative greedy algorithm.
\end{abstract}

\section{General Terms:}

Multiresolution, signal adaptive filter banks, iterative greedy algorithm.

\section{Keywords:}

Generalized triangular decoposition, pricipal component filter banks, iterative greedy algorithm, subband coder, multiresolution objective, singular value decomposition.

\section{INTRODUCTION}

In recent years, there has been a growing interest in desiging signal adaptive filter banks [1]. These filter banks, employed for subband coding and multiresolution objectives find applications in data compression, progressive transmission [2], [3], echo cancellation, etc. [4]. Based on generalized triangular decomposition (GTD) theory, a general family of perfect reconstruction filter banks adapted to their input statistics known as GTD filter banks was recently developed by Weng and Vaidyanathan [1].

The GTD filter banks are known to exist only in the two extremal cases. In the first case, the filters have zero memory. Here they reduce to GTD transform coders [5]. In the second case, the filter orders are unconstrained and they are as such unrealizable[1]. But these unrealizable GTD filter banks provide the upper bound on the achievable performance and also show a direction to proceed with the design of realizable signal adaptive filter banks with an improved performance.
These GTD filter banks are used as subband coders for optimizing the theoretical coding gain as shown in Fig. 1. In the subband coding objective, the optimal solutions can be obtained, if the suband signal satisfies both total decorrelation and spectrum equalization properties. These properties are satisfied, if the GTD filter bank is designed via the frequency dependent Geometric Mean Decompositin (GMD). Then the GTD filter bank is optimal in the coding gain sense and the optimal GTD subband coder [1] have superior performace than the optimal traditional subband coders in [6] and [7]. In this paper, we show that the GTD filter bank designed via the singular value decomposition (SVD) will reduce to principal component filter bank (PCFB) and gives optimal performance in the multiresolution objective. The finite impulse response (FIR) approximation problem of the optimal GTD filter banks is also done in this paper. The iterative greedy algorithm proposed in [8] is used for the FIR approximation of the desired paraunitary transfer function of unconstrained order.

This paper is organized as follows. In section II we briefly review the GTD theory and GTD filter banks. In section III we show the multiresolution optimality of GTD filter bank. In section IV we present the design of FIR GTD filter banks and section V provides numerical simulations. Section VI concludes the paper.

\section{REVIEW OF GTD THEORY AND GTD FILTER BANKS}

In this section, the theory of GTD [9] is brifely given and then we discuss the design of a GTD filter bank from a given input power spectral density (psd) matrix [1].

Definition: Generalized triangular decomposition (GTD): Let $\mathbf{H} \in$ $\boldsymbol{C}^{m \times n}$ be a rank-K matrix with singular values $\sigma_{h, 1}, \sigma_{h, 2}, \ldots \sigma_{h, k}$, in descending order. Let $r=\left[r_{1}, r_{2}, \ldots, r_{k}\right]$ be any vector which satisfies the multiplicative majorization property, i.e. the vector $\mathbf{h}$ multiplicatively majorizes a denoted as

$$
\mathbf{a} \prec_{\times} \mathbf{h},
$$

where $\mathbf{a}=\left[\left|r_{1}\right|,\left|r_{2}\right|, \ldots,\left|r_{K}\right|\right]$ and $h=\left[\sigma_{h, 1}, \sigma_{h, 1}, \ldots, \sigma_{h, k}\right]$. Then there exist matrices $\mathbf{R}, \mathbf{Q}$, and $\mathbf{P}$ such that $\mathbf{H}$ can be decomposed as

$$
\mathbf{H}=\mathbf{Q R P}^{\dagger}
$$

where $\mathbf{R}$ is a $K \times K$ upper triangular matrix with diagonal terms equal to $r_{k}, \mathbf{Q} \in \mathbf{C}^{m \times k}$ and $\mathbf{P} \in \mathbf{C}^{n \times k}$ both having orthonormal 


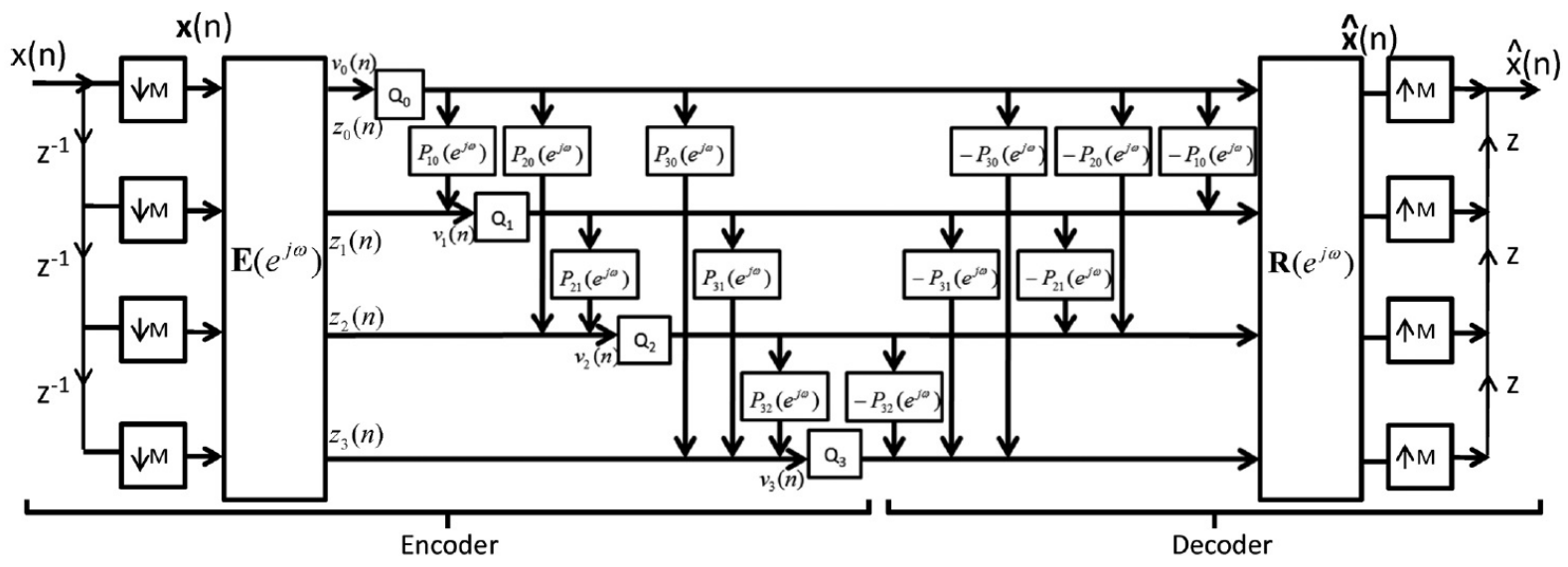

Fig. 1. The biorthogonal GTD subband coders for $M=4$ [1].

columns. Many existing orthogonal matrix decompositions, such as Singular Value Decomposition (SVD), QR decomposition, Geometric Mean Decomposition (GMD) are special cases of GTD.

By representing the estimation filters $P_{i k}\left(e^{j \omega}\right)$ from $k^{t h}$ stream to $i^{t h}$ stream of Fig. 1 by block $\mathbf{L}\left(e^{j \omega}\right)$ and replacing quantizers $Q_{i}$ by subband processors $S_{i}$, we can equivalently represent the GTD subband coder structure by the biorthogonal GTD filter bank as shown in Fig. 2. Here the vector process $\mathbf{x}(n)$ is assumed to be

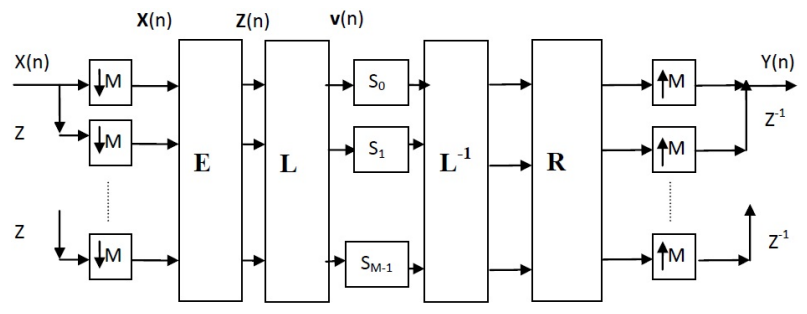

Fig. 2. The generalized triangular decomposition filter bank.

wide sense stationary (WSS) with psd matix $\mathbf{S}_{x x}\left(e^{j \omega}\right)$ and $\mathbf{E}\left(e^{j \omega}\right)$, $\mathbf{R}\left(e^{j \omega}\right)$ are the analysis and synthesis paraunitary polyphase matrices respectively, and $\mathbf{L}\left(e^{j \omega}\right)$ is the unit diagonal lower triangular matrix used to represent the frequency dependent prediction-based lower triangular transform (PLT) stage discussed in [10] and [11]. These blocks are obtained from the input psd matrix by performing the frequency dependent GTD [1] on the cholesky factor of the input $\operatorname{psd} \mathbf{S}_{x x}\left(e^{j \omega}\right)$ as

$$
\mathbf{S}_{x x}^{\dagger / 2}\left(e^{j \omega}\right)=\mathbf{Q}\left(e^{j \omega}\right) \mathbf{R}\left(e^{j \omega}\right) \mathbf{P}^{\dagger}\left(e^{j \omega}\right)
$$

then we can express

$$
\mathbf{S}_{\mathbf{x x}}\left(e^{j \omega}\right)=\mathbf{P}\left(e^{j \omega}\right) \mathbf{R}^{\dagger}\left(e^{j \omega}\right) \mathbf{Q}^{\dagger}\left(e^{j \omega}\right) \mathbf{Q}\left(e^{j \omega}\right) \mathbf{R}\left(e^{j \omega}\right) \mathbf{P}^{\dagger}\left(e^{j \omega}\right)
$$

Since $\mathbf{Q}\left(e^{j \omega}\right)$ is paraunitary, $\mathbf{Q}^{\dagger}\left(e^{j \omega}\right) \mathbf{Q}\left(e^{j \omega}\right)=\mathbf{I}$ for every $\boldsymbol{\omega}$. Consider a unit-diagonal lower triangular matrix $\mathbf{L}_{1}\left(e^{j \omega}\right)$ such that $\mathbf{R}^{\dagger}\left(e^{j \omega}\right)=\mathbf{L}_{\mathbf{1}}\left(e^{j \omega}\right) \operatorname{diag}\left(\left[\mathbf{R}_{\mathbf{1 1}}\left(e^{j \omega}\right), \ldots . \mathbf{R}_{\mathbf{M M}}\left(e^{j \omega}\right)\right]\right)$ and hence we can express $\mathbf{S}_{\mathbf{x x}}\left(e^{j \omega}\right)$ as (For simplicity, we drop the argument " $e^{j \omega ")}$

$$
\mathbf{S}_{\mathbf{x x}}=\mathbf{P L}_{1} \operatorname{diag}\left(\left[\mathbf{R}_{\mathbf{1 1}}^{2}, \mathbf{R}_{\mathbf{2} 2}^{2}, \ldots . \mathbf{R}_{\mathbf{M M}}^{2}\right]\right) \mathbf{L}_{\mathbf{1}}^{\dagger} \mathbf{P}^{\dagger} .
$$

So we can choose $\mathbf{E}\left(e^{j \omega}\right)=\mathbf{P}^{\dagger}\left(e^{j \omega}\right), \mathbf{R}\left(e^{j \omega}\right)=\mathbf{E}^{\dagger}\left(e^{j \omega}\right)=$ $\mathbf{P}\left(e^{j \omega}\right)$ and $\mathbf{L}\left(e^{j \omega}\right)=\mathbf{L}_{\mathbf{1}}^{-\mathbf{1}}\left(e^{j \omega}\right)$ and obtain the desired design blocks of GTD filter bank from the input psd. $S_{0}$ to $S_{M-1}$ are the subband processors, where $\mathrm{M}$ is the number of channels. $\downarrow \mathbf{M}$ denotes the decimators and $\uparrow \mathrm{M}$ denotes the interpolators with factor M.

\section{MULTIRESOLUTION OPTIMALITY OF GTD FILTER BANKS}

For a filter bank to be optimal in a multiresolution sense, it has to successively compact as much of the signal energy as possible into each subband starting with the first [12]. So far in the literature, the principal component filer bank (PCFB) is known to be optimal in the multiresolution optimality criteria, since the subband signal of PCFB satisfies the spectrum majorization and spectrum decorrelation properties, and compacts as much signal energy as possible into the first few subbands. If the GTD filter bank is designed via singular value decomposition then the GTD also satisfies the spectrum majorization and spectrum decorrelation properties. In this case, the block $\mathbf{L}\left(e^{j \omega}\right)$ in Fig. 2 is an identity matrix and includes PCFB as a special case of GTD filter bank.

In order to assess the performance of the GTD filter bank for multiresolution objectives, one suitable measure is the proportion of the partial subband variances to the total [8]. By preserving only $\mathrm{L}$ out of $\mathrm{M}$ subbands, this proportion is given by

$$
P(L) \triangleq \frac{\left(\sum_{k=0}^{L-1} \sigma_{w_{k}}^{2}\right)}{\left(\sum_{k=0}^{M-1} \sigma_{w_{k}}^{2}\right)}, \quad 1 \leq L \leq M
$$

where $\sigma_{w_{k}}^{2}$ is the subband variance of the $k^{\text {th }}$ channel. Since the GTD filter bank (designed via the frequency dependent SVD) satisfies subband majorization property, the GTD filter bank maximizes $\mathrm{P}(\mathrm{L})$ for all $\mathrm{L}$.

Hence in addition to being optimal for coding gain, the GTD filter bank also provides optimal performance with respect to multiresolution optimality criteria, if we design the GTD filter bank via SVD. 


\section{FIR APPROXIMATION OF GTD FILTER BANKS}

In this section, the FIR approximation of GTD filter bank is discussed. Here the objective is to minimize the weighted meansquared Frobenius norm error between the desired paraunitary system $\mathbf{D}\left(e^{j \omega}\right)$ and FIR paraunitary system $\mathbf{F}\left(e^{j \omega}\right)$ responses given by [8].

$$
\xi \triangleq \frac{1}{2 \pi} \int_{0}^{2 \pi} \mathbf{W}(\omega)\left\|\mathbf{D}\left(e^{j \omega}\right)-\mathbf{F}\left(e^{j \omega}\right)\right\|_{F}^{2} d \omega .
$$

where, $\mathbf{W}(\omega)$ is a weight function. The FIR paraunitary system $\mathbf{F}\left(e^{j \omega}\right)$ is to be parameterized in terms of Householder-like degreeone building blocks with McMillan degree (N-1) of the form [13]

$$
\mathbf{F}(z)=\mathbf{V}(z) \mathbf{U}
$$

where $\mathbf{U}$ is a some $p \times r$ unitary matrix and $\mathbf{V}(\mathrm{z})$ is a $p \times p$ paraunitary matrix consisting of $(\mathrm{N}-1)$ degree-one Householder-like building blocks of the form

$$
\begin{gathered}
\mathbf{V}(z)=\prod_{i=N-1}^{1} \mathbf{V}_{i}(z) \\
\mathbf{V}_{i}(z)=\mathbf{I}_{p}-\mathbf{v}_{i} \mathbf{v}_{i}^{\dagger}+z^{-1} \mathbf{v}_{i} \mathbf{v}_{i}^{\dagger}, \quad 1 \leqslant i \leqslant N-1
\end{gathered}
$$

Here $\mathbf{v}_{i}$ are the unit norm vectors and $\mathbf{I}_{p}$ is $\mathrm{p} \times \mathrm{p}$ identity matrix. Since there is no direct closed form solutions available for the parameters $\left\{\mathbf{v}_{k, o p t}\right\}$ and $\mathbf{U}_{\text {opt }}$ of optimal FIR approximant $\mathbf{F}_{\text {opt }}(z)$, we have to go for the optimization algorithms to find the optimal FIR approximant $\mathbf{F}_{\text {opt }}(z)$, which gradually decreases the mean squared error $\xi$ as a function of iterations. In [8] it is shown that using the iterative greedy algorithm the optimal FIR approximant is obtained by optimizing one set of parameters in closed form, assuming that all the other parameters are fixed. In this paper, we are using the iterative greedy algorithm to find the FIR approximant, since in iterative greedy algorithm, the observed mean squared error is guaranteed to not increase at each iteration.

The design procedure for FIR GTD filter banks is given below.

Design of FIR GTD Filter Banks:

(1) Obtain the cholesky factor of input $\operatorname{psd} \mathbf{S}_{x x}\left(e^{j \omega}\right)$.

(2) Perform the frequency dependent GTD on cholesky factor $\mathbf{S}_{x x}^{\dagger / 2}\left(e^{j \omega}\right)$ of input psd matrix $\mathbf{S}_{x x}\left(e^{j \omega}\right)$, i.e. $\mathbf{S}_{x x}^{\dagger / 2}\left(e^{j \omega}\right)=$ $\mathbf{Q}\left(e^{j \omega}\right) \mathbf{R}\left(e^{j \omega}\right) \mathbf{P}^{\dagger}\left(e^{j \omega}\right)$.

(3) Find the optimal FIR approximant $\mathbf{F}_{\text {opt }}\left(e^{j \omega}\right)$ which approximates the desired response $\mathbf{D}\left(e^{j \omega}\right)=\mathbf{P}\left(e^{j \omega}\right)$, by using the iterative greedy algorithm [14], so that $\mathbf{E}\left(e^{j \omega}\right)=\mathbf{F}^{\dagger}\left(e^{j \omega}\right)$, and $\mathbf{R}\left(e^{j \omega}\right)=\mathbf{F}\left(e^{j \omega}\right)$.

(4) Compute the LDU decompostion (where 'LDU' stands for 'Lower Diagonal Upper' triangular matrices):

$\mathbf{E}\left(e^{j \omega}\right) \mathbf{S}_{x x}\left(e^{j \omega}\right) \mathbf{E}^{\dagger}\left(e^{j \omega}\right)=\mathbf{L}_{1}\left(e^{j \omega}\right) \mathbf{D}\left(e^{j \omega}\right) \mathbf{L}_{1}^{\dagger}\left(e^{j \omega}\right)$, and take the estimation fiters as $\mathbf{L}\left(e^{j \omega}\right)=\mathbf{L}_{1}^{-1}\left(e^{j \omega}\right)$.

Construct the FIR GTD filter bank as in Fig.1, using the desired design blocks obtained from the design steps presented above.

\section{SIMULATION RESULTS}

In this section we present the simulation results of FIR GTD filter bank in the multiresloution and coding gain objectives.

\subsection{Multiresolution Optimality Results}

The FIR approximation results of the GTD filter banks, by using the iterative greedy algorithm to aproximate the synthesis polyphase matrices $\mathbf{R}\left(e^{j \omega}\right)$ are shown in Fig. 3. Here the GTD FB is designed via the frequency dependent SVD for the input of AR(4) process, with $\mathrm{M}=4$ and $\mathrm{N}=10$. Iterative greedy algorithm is run for 1000 iterations, and the mean squared error obtained is $\xi=0.7190$. Fig. 3 shows a good magnitude fit to the desired response by using iterative greedy algorithm. In Fig. 4 the performances of GTD transform coder (GTD TC), unconstrained order GTD filter bank (GTD FB), and FIR GTD filter bank (FIR GTD FB) are compared. The realizable FIR GTD filter bank gives the performance between those of GTD transform coder and unconstrained order GTD filter bank.

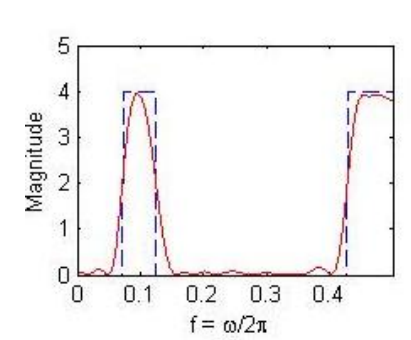

(a)

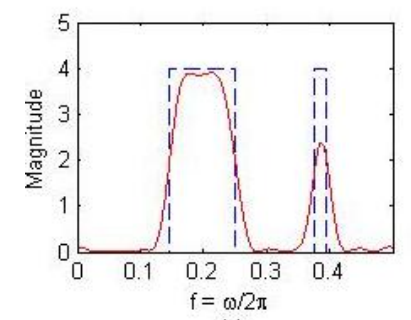

(c)

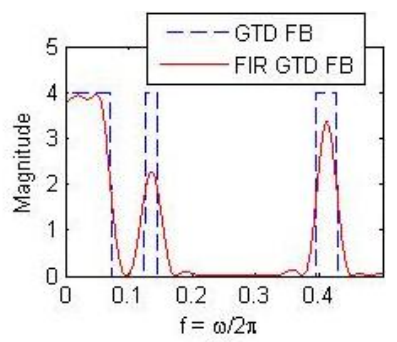

(b)

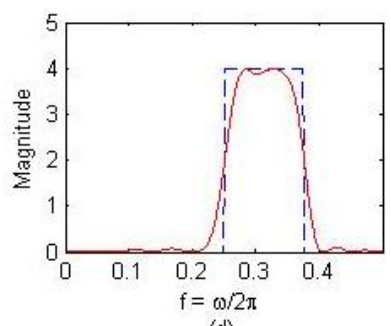

(d)
Fig. 3. The synthesis filter magnitude squared response of unconstrained order and FIR GTD filter bank: (a) to (d) are the synthesis filter magnitude squared responses of channel 0 to 3 .

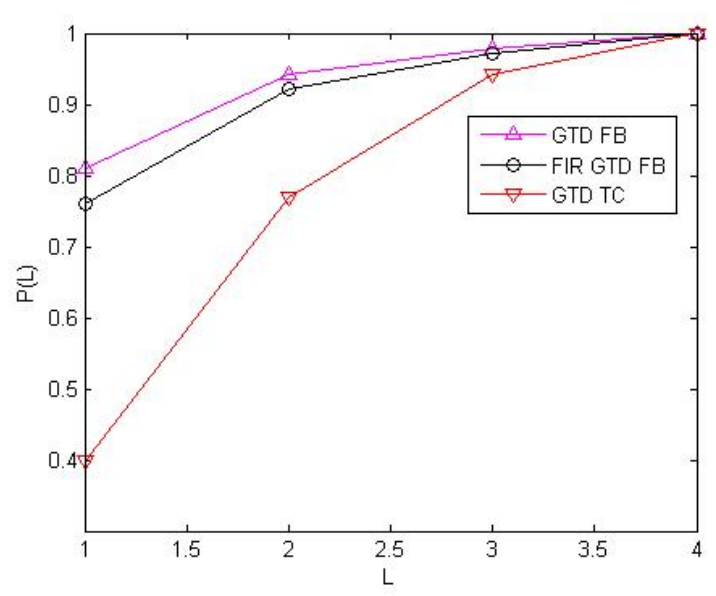

Fig. 4. Proportion of the total variance $\mathrm{P}(\mathrm{L})$ as a function of the number of subbands kept $\mathrm{L}$, for an $\mathrm{M}=4$ channel system with $\mathrm{N}=10$. 


\subsection{Coding Gain Results}

In the subband coding objective, the optimal solutions can be obtained, if the subband signal satisfies both total decorrelation and spectrum equalization properties [1]. These properties are satisfied, if the GTD FB is designed via the frequency dependent GMD. Then the FB is optimal in the coding gain sense. The coding gain is given by

$$
\mathbf{G}_{C}=\frac{\left.\int_{0}^{2 \pi} \frac{1}{M} \operatorname{Tr}\left(\mathbf{S}_{x x}\left(e^{j \omega}\right)\right)\right) d \omega}{\left(\prod_{i=0}^{M-1} \int_{0}^{2 \pi} \mathbf{S}_{v_{i}}\left(e^{j \omega}\right) d \omega\right)^{\frac{1}{M}}}
$$

The performance of the FIR GTD filter Bank is compared with that of the unconstrained order filter bank, and also with the GTD transform coder. Here AR(2) process is used to model the input psd for GTD filter Bank with poles at $z_{ \pm}=\rho e^{ \pm j \theta}$. The simulation results are shown in Fig. 5. The coding gain is shown by varying $\rho$ from 0.95 to 0.99 with $\theta=\frac{\pi}{5}$, and $\mathrm{M}=4$, and it is observed that for $\mathrm{N}=10$, FIR GTD filter bank shows a performance very close to that of the unconstrained order GTD filter bank.

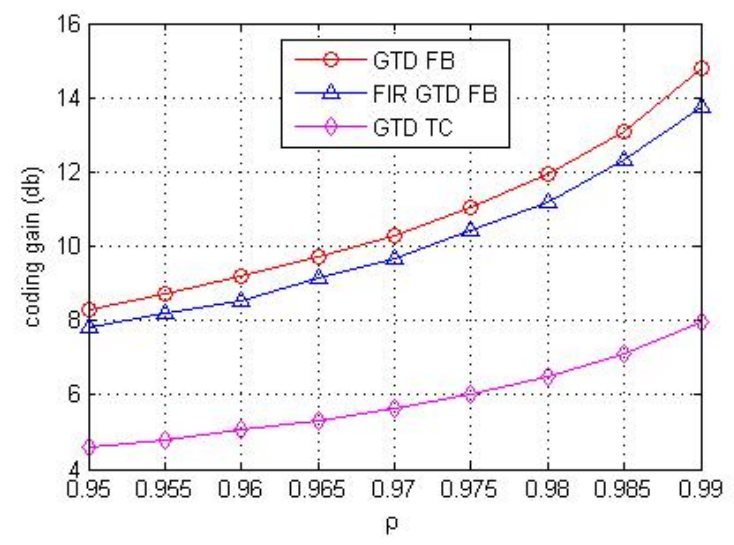

Fig. 5. Coding gain of GTD filter bank as a function of $\rho$ from 0.95 to 0.99 , with $\mathrm{M}=4$ and $\theta=\frac{\pi}{5}$ for $\mathrm{AR}(2)$ process.

\section{CONCLUSION}

The GTD filter bank is reported to be used as a subband coder for optimizing the coding gain. In this paper we have shown that the GTD filter bank designed via SVD will be reduced to the PCFB and gives optimal performance in the multiresolution objective. The FIR approximation of the paraunitary blocks of GTD filter bank is also done by using iterative greedy algorithm.

\section{REFERENCES}

[1] C. C. Weng, and P. P. Vaidyanathan, "The Role of GTD in Optimizing Perfect Reconstruction Filter Banks," IEEE Trans. Signal Process., vol. 60, pp. 112118, Jan. 2012.

[2] S. Akkarakaran and P. P. Vaidyanathan, "Filterbank optimization with convex objectives and the optimality of principal component forms," IEEE Trans. Signal Process., vol. 49, no. 1, pp. 100114, Jan. 2001.

[3] Sheeba V S, Elizabeth Elias, "Design of Two-Dimensional Signal Adapted Filter Banks for Application in Image Processing," WSEAS Transactions on Signal Processing Issue 9, Vol. 2, Sept. 2006, pp 1281-1286.
[4] Elizabeth Elias, Sheeba V S, and Anand R, "FIR Principal Component Filter Banks in Echo Cancellation," CSI Journal, Young Horizon-Computing and Informatics, Vol. 1, No. 1, Nov. 2006, pp 28-31.

[5] C. C. Weng, C. Y. Chen, and P. P. Vaidyanathan, "Generalized triangular decomposition in transform coding," IEEE Trans. Signal Process., vol. 58, no. 2, pp. 566574, Feb. 2010.

[6] P. P. Vaidyanathan,"Theory of optimal orthonormal subband coders," IEEE Trans. Signal Process., vol. 46, pp. 15281543, Jun. 1998.

[7] P. P. Vaidyanathan,"Theory of optimal orthonormal subband coders," IEEE Trans. Signal Process., vol. 46, pp. 15281543, Jun. 1998.

[8] A. Tkacenko and P. P. Vaidyanathan, "Iterative greedy algorithm for solving the FIR paraunitary approximation problem," IEEE Trans. Signal Process., vol. 54, pp. 146160, Jan. 2006.

[9] Y. Jiang, W. W. Hager, and J. Li, "Generalized triangular decomposition," Math. Computat., Oct. 2007.

[10] S. M. Phoong and Y. P. Lin, "MINLAB: Minimum noise structure forladder-based biorthogonal filter banks," IEEE Trans. Signal Process., vol. 48, no. 2, pp. 465476, Feb. 2000.

[11] S. M. Phoong and Y. P. Lin, "Prediction-based lower triangular transform," IEEE Trans. Signal Process., vol. 48, no. 7, pp. 19471955, Jul. 2000.

[12] M. K. Tsatsanis and G. B. Giannakis,"Principal component filter banks for optimal multiresolution analysis," IEEE Trans. Signal Process., vol. 43, no. 8, pp. 17661777, Aug. 1995.

[13] P. P. Vaidyanathan, Systems and Filter Banks. Prentice-Hall, 1993.

[14] A. Tkacenko. Matlab m-Files. [Online]. Available: http://www.systems.caltech.edu/dsp/students/andre/index.html 